\title{
LAS PÍLDORAS EDUCATIVAS: SU VALORACIÓN POR LOS ESTUDIANTES DE GRADO DE LA UNIVERSIDAD DE LEÓN
}

\author{
María José Fínez Silva \\ Universidad de León \\ mjfins@unileon.es \\ M. Consuelo Morán Astorga \\ Universidad de León \\ mcmora@unileon.es \\ Gema Vallejo Pérez \\ Universidad de León \\ gvalp@unileon.es
}

Recepción Artículo: 13 octubre 2021

Admisión Evaluación: 13 octubre 2021

Informe Evaluador 1: 13 octubre 2021

Informe Evaluador 2: 14 octubre 2021

Aprobación Publicación: 14 octubre 2021

\section{RESUMEN}

En este trabajo nos planteamos como objetivo principal evaluar la implementación de una innovadora metodología docente: las Píldoras Educativas. Se aplicó esta metodología en dos asignaturas del Grado en Relaciones Laborales y Recursos Humanos de la Universidad de León. Participaron en la evaluación 30 estudiantes, con una media de edad de 20 años, que respondieron al Cuestionario de Evaluación de las Píldoras Educativas, elaborado ad hoc. Según los resultados, el 96\% de los estudiantes valoraron que las Píldoras Educativas facilitaron "bastante o mucho" la comprensión de los contenidos, que aprendieron mejor con esta metodología y que les resultó más motivadora a la hora de estudiar. El 4\% restante valoró con la opción "poco" los aportes de la Píldoras Educativas para la comprensión, el aprendizaje y la motivación. Respecto a la pregunta de si consideraron a las Píldoras Educativas mejor metodología que la tradicional, un 93\% indicó que "bastante o mucho" y un 6\% valoró que esta metodología no era mejor que la tradicional. Se hallaron diferencias significativas en función del sexo en comprensión y aprendizaje. Las mujeres valoraron en mayor medida que los varones a las Píldoras Educativas como recurso eficaz a la hora de comprender los contenidos y aprenderlos. Concluimos que las Píldoras Educativas son un recurso facilitador del aprendizaje y de la motivación en los estudiantes, que tienen la ventaja de la condensación de los contenidos, del atractivo del formato, que al ser online e integrado en las plataformas digitales dan la posibilidad de visualizarlas repetidamente y en distintas situaciones. Como mejoras posibles de esta investigación consideramos que se deberá en el futuro implementar esta metodología en más asignaturas y con más estudiantes.

Palabras clave: píldoras educativas; metodología docente; aprendizaje; motivación; estudiantes universitarios 


\section{LAS PÍLDORAS EDUCATIVAS: SU VALORACIÓN POR LOS ESTUDIANTES DE GRADO DE LA UNIVERSIDAD DE LEÓN}

\section{ABSTRACT}

Educational pills: how they are valued by undergraduate students at the university of Leon. The main objective of the present paper is to assess the implementation of an innovative teaching methodology known as Learning Pills. This methodology has been applied to two subjects of the Bachelor of Labour Relations and Human Resources at the University of León.

Thirty students participated in the project, the average age for this group being 20 years old. The students were asked to fill in an evaluation questionnaire on learning pills, developed on an ad-hoc basis. According to the results, $96 \%$ of students deemed the learning pills as "quite" or "very" helpful for understanding the subject contents, describing them as highly motivational and learning-facilitating. The remaining $4 \%$, judged the learning pills to have contributed "little" to their understanding, their learning, and their motivation.

When asked whether they considered learning pills to be a better methodology than the traditional one, 93\% of students judged the pills to be so, whereas a $6 \%$ considered the traditional methodology a better option.

Significant differences in understanding and learning contents were found based on the students' gender. Women deemed learning pills to be an effective resource for learning in a higher level than men did.

In summary, the study concludes that learning pills are a facilitator of both learning and student motivation thanks to the advantages they offer in condensing contents, their appealing format, and their suitability to be integrated into digital platforms, which allows students to easily access them when needed. As to potential improvements for this research, in the future this methodology should be implemented in further subjects and to more students.

Keywords: learning pills; teaching methodology; learning; motivation; university students

\section{INTRODUCCIÓN}

La sociedad actual necesita más recursos tecnológicos para dar respuesta a las nuevas demandas de aprendizaje. Los recursos tecnológicos actuales son muy potentes y permiten la exposición a los mismos de manera reiterada, sin necesidad de horarios fijos, ni de lugares establecidos. Permiten que los estudiantes se acerquen a Ios contenidos objeto del aprendizaje en cualquier momento y en cualquier lugar. Esto da más flexibilidad al estudiante a la hora de interaccionar con ellos (Sánchez, 2012).

Los cambios sociales y tecnológicos recientes demandan y permiten la inmediatez en el acceso a la información. Esta oportunidad la podemos aprovechar para innovar en la metodología docente.

Debemos tener en cuenta, además del cambio tecnológico, que las universidades españolas están inmersas en el Espacio Europeo de Educación Superior (EEES), que exige a los profesores aunar la actualidad tecnológica con las necesidades académicas. Dentro de las directrices del EEES es especialmente interesante el nuevo paradigma de enseñanza-aprendizaje que centra sus esfuerzos en que el estudiante sea capaz de manejar el conocimiento, seleccionarlo, actualizarlo y aprenderlo. Se trata de que el estudiante adquiera más compromiso y protagonismo en su propia capacidad de aprender, es decir, que adopte un papel más activo en su propio aprendizaje (Morán y Fínez, 2019).

Ya en el panorama actual, la situación social provocada por la aparición del COVID-19 ha acelerado la sustitución de parte de la educación presencial por una educación online, requiriendo a los profesores a utilizar los recursos tecnológicos a través de plataformas digitales. Esto coloca a los profesores universitarios en un escenario obligatorio donde poner a prueba su adaptación a los nuevos recursos. Las Píldoras Educativas se proponen como uno de los nuevos recursos a utilizar en el contexto de la Educación Superior.

Las Píldoras Educativas son lecciones breves de temas concretos que se graban en formato digital y se suben a una plataforma online con el objetivo de que los estudiantes puedan acceder a ellas cuando lo deseen. Colomo y Aguilar (2017) consideran la Píldora Educativa como la unidad más pequeña de información dotada de significado. Su objetivo es enseñar y reforzar conceptos concretos, captando la atención del estudiante, exponiendo Ios contenidos de una manera atractiva, dinámica e interactiva. A pesar de su brevedad, implican un análisis profundo de la materia. Este recurso educativo está diseñado para complementar las estrategias tradicionales de 
enseñanza y así facilitar la comprensión de los conceptos (Abad y Hernández-Ramos, 2017). Pueden usarse de forma independiente para tratar un tema específico, o como un conjunto de Píldoras Educativas complementarias para profundizar en un tema más general (Colomo y Aguilar, 2017).

El formato de las Píldoras Educativas es digital y audiovisual. Se pueden encontrar como videotutoriales, podcast, infografías, o exposiciones del profesor apoyadas con presentaciones. Independientemente del tipo, todas ellas se almacenan en una plataforma online donde los estudiantes pueden acceder fácilmente. Hay evidencia de la influencia positiva de estas nuevas experiencias para profesores y estudiantes (Woods, 2020). Las Píldoras Educativas se diseñan teniendo en cuenta las necesidades de los estudiantes, buscando el compromiso con ellos para seguir este nuevo método.

Las Píldoras Educativas son un recurso atractivo e innovador que motiva a los estudiantes, nativos digitales, muy familiarizados con el formato online. Se caracterizan por ser de corta duración, entre 10 y 15 minutos, y se centran en un solo tema, o concepto, desarrollando un contenido muy específico. El contenido de las Píldoras Educativas es muy condensado y expone los puntos esenciales a desarrollar por el profesor. Según Yokoyama (2019) las Píldoras Educativas amplían los procesos de aprendizaje desarrollados en el aula. La temática que pueden tratar es muy amplia, actualmente se están implementando en todos los campos del conocimiento, desde las ingenierías hasta el aprendizaje de idiomas (Crespo y Sánchez-Saus, 2020).

Se busca que los contenidos de las Píldoras Educativas sean aplicables y prácticos, es decir, que haya transferencia a la realidad. Otra característica es que pueden ser visionadas de forma repetida, a demanda del estudiante, a través de diferentes dispositivos de fácil acceso como el ordenador o el teléfono móvil con conexión a internet (Salinas y Marín, 2014). Así se amplían los escenarios de aprendizaje porque se pueden visualizar en cualquier espacio y momento, no necesariamente en el aula. El estudiante tiene autonomía para volver a ver el contenido accediendo a la plataforma donde está alojado. La posibilidad de poder ver las Píldoras Educativas tantas veces como deseen facilita la preparación de los contenidos a modo de repaso, o para afianzarlos de cara a la evaluación (Crespo y Sánchez-Saus, 2020). Además, se adaptan a los ritmos de cada estudiante de forma que éste se convierte en sujeto activo y protagonista de su propio aprendizaje (Rodríguez-García, Hinojo-Lucena y Ágreda-Montoro, 2017).

\section{OBJETIVOS DE LA INVESTIGACIÓN}

Los objetivos de este trabajo fueron tres, aprender a elaborar Píldoras Educativas como recurso didáctico, implementar las mismas como una nueva metodología en dos asignaturas de la Facultad de Ciencias del Trabajo de la Universidad de León y evaluar si los estudiantes las consideran útiles para mejorar la comprensión y el aprendizaje de los contenidos, así como para aumentar la motivación en la propia asignatura.

\section{MUESTRA Y/O PARTICIPANTES}

Los participantes de esta investigación fueron 30 estudiantes de las asignaturas de Psicología del Trabajo y Orientación Laboral y Empleo del Grado en Relaciones Laborales y Recursos Humanos de la Universidad de León. La media de edad fue 20,90 con una desviación típica de 1,77. El 54\% fueron mujeres.

\section{METODOLOGÍA Y/O INSTRUMENTOS UTILIZADOS}

Para aplicar las Píldoras Educativas se utilizaron dos modalidades, en una de ellas la profesora grabó las Píldoras Educativas y las puso a disposición de los estudiantes en la plataforma Moodle, donde los estudiantes pudieron visualizarlas tantas veces como necesitaron. Los estudiantes vieron las Píldoras Educativas antes de ir a la clase. La otra modalidad consistió en que la profesora, al iniciar la clase, hacía la presentación en vivo de las Píldoras Educativas con el contenido del tema muy condensado. En ambos casos, una vez vistas las Píldoras Educativas se pasaba a la parte práctica de la clase.

El instrumento utilizado fue el Cuestionario de Evaluación de las Píldoras Educativas, elaborado por las propias investigadoras. Consta de cuatro ítems que se respondieron en una escala tipo Likert con tres opciones de 


\section{LAS PÍLDORAS EDUCATIVAS: SU VALORACIÓN POR LOS ESTUDIANTES DE GRADO DE LA UNIVERSIDAD DE LEÓN}

respuesta 1 (poco), 2 (bastante) y 3 (mucho). Las preguntas que debían contestar los estudiantes eran "Las Píldoras Educativas te facilitan la comprensión de los contenidos de la asignatura", "Crees que aprendes mejor con esta metodología", "Te resulta más motivadora esta nueva metodología" y "Crees que esta metodología es mejor que la tradicional".

El cuestionario fue aplicado por las propias investigadoras en el aula al finalizar las asignaturas durante el segundo semestre del curso 2020-2021. Los datos fueron analizados con el programa estadístico SPSS versión 26. Las pruebas que se realizaron fueron análisis descriptivos, de frecuencias y la prueba $t$ de diferencia de medias.

\section{RESULTADOS ALCANZADOS}

Los resultados que se muestran en la Tabla 1 indican que el $96,6 \%$ de los estudiantes valoró que las Píldoras Educativas les facilitaron mucho o bastante la comprensión de los contenidos de la asignatura. Solamente un alumno (3,3\%) valoró que las Píldoras Educativas le facilitaron poco la comprensión de los contenidos.

En cuanto a si aprendieron mejor con esta metodología, un 96,7\% valoraron que mucho o bastante y un 3,3\% valoró como poco el aporte de las Píldoras Educativas en la mejora de su aprendizaje.

Un tercer aspecto evaluado fue si las Píldoras Educativas resultaban motivadoras. El 96,6\% de los estudiantes indicaron que mucho o bastante. 2 estudiantes (6,7\%) consideraron poco motivadoras las Píldoras Educativas.

Respecto a si valoraban que la metodología de las Píldoras Educativas era mejor que la tradicional, un 93\% indicaron que bastante 0 mucho, y un $6 \%$ informaron que esta metodología no era mejor que la tradicional.

Tabla 1.

Frecuencias y porcentajes en comprensión, aprendizaje, motivación y metodología

\begin{tabular}{lcccccccc}
\hline & \multicolumn{2}{c}{ COMPRENSIÓN } & \multicolumn{2}{c}{ APRENDIZAJE } & \multicolumn{2}{c}{ MOTIVACIÓN } & \multicolumn{2}{c}{ MEJOR METODOLOGÍA } \\
\hline Escala & Frecuencia & Porcentaje & Frecuencia & Porcentaje & Frecuencia & Porcentaje & Frecuencia & Porcentaje \\
\hline Poco & 1 & 3,3 & 1 & 3,3 & 1 & 3,3 & 2 & 6,7 \\
\hline Bastante & 10 & 33,3 & 17 & 56,7 & 13 & 43,3 & 9 & 30 \\
\hline Mucho & 19 & 63,3 & 12 & 40,0 & 16 & 53,3 & 19 & 63,3 \\
\hline
\end{tabular}

También fue objetivo de este estudio analizar si existían diferencias significativas en función del sexo y del grupo. Según se aprecia en la Tabla 2 existen diferencias significativas en función del sexo en comprensión ( $t=$ $-3,029 ; p=, 006)$ y aprendizaje $(t=-2,481 ; p=, 021$,$) a favor de las mujeres.$

No se hallaron diferencias significativas en función del grupo. Entendiendo por grupo los estudiantes que cursaban las asignaturas en las que se realizó el estudio que fueron "Psicología del Trabajo" y "Orientación Laboral y Empleo". 
Tabla 2.

Diferencias en función del sexo en comprensión, aprendizaje, motivación y metodología

\begin{tabular}{|c|c|c|c|c|c|c|c|c|}
\hline & \multicolumn{2}{|c|}{$\begin{array}{l}\text { Prueba de } \\
\text { Levene de } \\
\text { igualdad de } \\
\text { varianzas }\end{array}$} & \multirow[b]{2}{*}{$t$} & \multirow[b]{2}{*}{$\mathrm{gl}$} & \multicolumn{3}{|c|}{ Prueba $t$ para la igualdad de medias } & \multirow[b]{2}{*}{ D.T } \\
\hline & $\mathrm{F}$ & Sig. & & & Sig. Bilat. & Sexo & Media & \\
\hline \multirow{2}{*}{ Comprensión } & \multirow{2}{*}{2,019} & \multirow{2}{*}{, 168} & \multirow{2}{*}{$-3,029$} & \multirow{2}{*}{24} & \multirow{2}{*}{, 006} & Varón & 2,20 & ,632 \\
\hline & & & & & & Mujer & 2,81 &, 403 \\
\hline \multirow{2}{*}{ Aprendizaje } & \multirow{2}{*}{8,308} & \multirow{2}{*}{, 008} & \multirow{2}{*}{$-2,481$} & \multirow{2}{*}{24} & \multirow{2}{*}{, 021} & Varón & 2,00 & 471 \\
\hline & & & & & & Mujer & 2,50 &, 516 \\
\hline \multirow{2}{*}{ Motivación } & \multirow{2}{*}{,662 } & \multirow{2}{*}{, 424} & \multirow{2}{*}{$-1,125$} & \multirow{2}{*}{24} & \multirow{2}{*}{,272 } & Varón & 2,30 & 675 \\
\hline & & & & & & Mujer & 2,56 &, 512 \\
\hline \multirow{2}{*}{ Mejor Metodología } & \multirow{2}{*}{6,428} & \multirow{2}{*}{, 018} & \multirow{2}{*}{,- 858} & \multirow{2}{*}{24} & \multirow{2}{*}{,399 } & Varón & 2,40 & 843 \\
\hline & & & & & & Mujer & 2,63 &, 500 \\
\hline
\end{tabular}

\section{DISCUSIÓN Y CONCLUSIONES}

Las Píldoras Educativas se caracterizan por su condensación, mayor impacto, fácil accesibilidad y eficacia para comprender mejor los contenidos de las asignaturas. Respecto a la elaboración de las Píldoras Educativas podemos concluir que el resultado ha sido satisfactorio, pero podría ser mejorable si los profesores hubieran dispuesto de programas de edición de vídeo que mejoraran el resultado final de las grabaciones, así como de cámaras de mayor calidad para captar mejor la imagen y el audio. Elaborar las Píldoras Educativas supuso un esfuerzo añadido para las profesoras, pero con una repercusión positiva en los estudiantes. Las Píldoras Educativas elaboradas tenían las siguientes características: se centraron en un tema concreto, sintetizaban perfectamente los contenidos, tenían una duración acotada, entre 10 y 15 minutos, y los contenidos eran aplicables y prácticos.

La implementación de las Píldoras Educativas se realizó en dos modalidades. En un grupo la profesora grabó las Píldoras Educativas en un vídeo explicativo apoyado por una presentación power point y lo puso a disposición de los estudiantes en la plataforma Moodle. A los estudiantes se les dio la indicación de que vieran las Píldoras Educativas antes de asistir a la clase. La otra modalidad consistió en que la profesora expuso de forma presencial las Píldoras Educativas al inicio de la clase. Independientemente de la modalidad, la estructura, el formato y la duración de las Píldoras Educativas fueron equiparables.

Una vez vistas las Píldoras Educativas las profesoras matizaron los contenidos y dieron paso a la parte práctica. Con esta metodología, se permitió avanzar en los conceptos teóricos para dedicar más tiempo a la parte práctica, la clase fue más ágil y se optimizó el tiempo. Los estudiantes acogieron bien este sistema y la mayoría acudía a las clases con las Píldoras Educativas vistas previamente.

Las Píldoras Educativas se pudieron visualizar de forma repetida, y en cualquier contexto utilizando dispositivos electrónicos de fácil manejo como son el móvil, la tablet o el ordenador con conexión a internet. Los escenarios de aprendizaje se ampliaron, ya no solo era el aula el único contexto. Eines y cols. (2018) ponen en relieve el uso de nuevos contextos de aprendizaje y la utilización de plataformas digitales como nuevo espacio educativo del siglo XXI. Las Píldoras Educativas son un recurso que se puede utilizar para aprender, afianzar los conocimientos, facilitar el repaso y preparar los exámenes debido a la gran síntesis de los conceptos relevantes propios de cada tema y a su corta duración.

En cuanto a la evaluación que los estudiantes hicieron de las Píldoras Educativas, podemos concluir que son un recurso didáctico eficaz en el desarrollo de las clases, estos resultados van en la línea de los obtenidos por Bustamante y cols. (2016) que en una muestra de estudiantes universitarios de Zaragoza halló que las Píldoras Educativas eran un recurso útil en el aula que motivaba a los estudiantes y potenciaba el aprendizaje. Las profesoras hicieron un esfuerzo importante por condensar lo más relevante de los temas y lo plasmaron en una gra- 


\section{LAS PÍLDORAS EDUCATIVAS: SU VALORACIÓN POR LOS ESTUDIANTES DE GRADO DE LA UNIVERSIDAD DE LEÓN}

bación, o lo expusieron en clase, por lo que esa síntesis también facilitó la comprensión de lo esencial. Al facilitar la comprensión, se potenció el aprendizaje posterior, los estudiantes interiorizaron de una forma más sencilla los conceptos. Estos resultados son similares a los hallados por Muñoz y Cols. (2016). Otro aspecto positivo de las Píldoras Educativas fue su formato digital afín a los medios tecnológicos que los estudiantes manejan habitualmente, lo valoraron como más motivador por la forma de recibir los contenidos. Crespo y Sánchez-Saus (2020) hallaron que las Píldoras Educativas no sólo son eran un recurso de estudio, sino también motivador del mismo. Guevara, Sánchez-Moro y Barrientos (2020) hallaron que al utilizar la Píldoras Educativas los estudiantes valoraban que la motivación por la asignatura era mayor, que se había favorecido el buen clima en el aula y que habían comprendido mejor los contenidos.

En cuanto a la comparación de las Píldoras Educativas con la metodología tradicional, los estudiantes informaron de que preferían la nueva metodología en un porcentaje muy elevado. En este estudio se combinaron las Píldoras Educativas con la metodología tradicional y el resultado ha sido positivo. Carballido-Landeira (2020) halla resultados afines en una investigación con estudiantes de Ingeniería Química.

En esta investigación no se hallaron diferencias en función del grupo, pero sí del sexo. Las mujeres valoraron en mayor medida que los varones las Píldoras Educativas como recurso eficaz para comprender mejor los contenidos y aprenderlos.

Como aspectos a mejorar en futuras investigaciones, habría que dotar a los docentes de programas de edición de vídeo que mejorasen el resultado final de las grabaciones. También mentalizar a los estudiantes de que deben acudir a la clase con las Píldoras Educativas vistas previamente porque de lo contrario no siguen adecuadamente la dinámica de la clase.

\section{REFERENCIAS BIBLIOGRÁFICAS}

Abad, F. M., y Hernández-Ramos, J. P. (2017). Flipped Classroom con píldoras audiovisuales en prácticas de análisis de datos para la docencia universitaria: percepción de los estudiantes sobre su eficacia. En S. Pérez, G. Castellano y A. Pina (Coords.), Propuesta de Innovación Educativa en la Sociedad de la Información, pp. 92105. Eindhoven, NL: Adaya Press.

Bustamante, J.C., Larraz, N, Sánchez, E., Carrón, J., Antoñanzas, J. L. y Salavera, C. (2016). El uso de las píldoras formativas competenciales como experiencia de innovación docente en el grado de magisterio en Educación Infantil. ReiDoCrea: Revista Electrónica de Investigación y Docencia Creativa, 5, 223-236.

Carballido-Landeira, J. (2020). Uso de píldoras audiovisuales como soporte del conocimiento y ayuda del aprendizaje. En Redine (Ed.) Conference proceedings CIVINEDU 2020: 4th International Virtual Conference on Educational Research and Innovation (pp. 220-222). Madrid: Adaya Press.

Colomo, E., y Aguilar, A. I. (2017). Píldoras formativas en la educación online: posibilidades y limitaciones. En J. Ruiz-Palmero, J. Sánchez-Rodríguez, J. y E. Sánchez-Rivas (Edit.). Innovación Docente y uso de las TIC en Educación, (pp. 1-10). Málaga: UMA Editorial.

Crespo, M. y Sánchez-Saus, M. (2020). Píldoras formativas para la mejora educativa universitaria: el caso del Trabajo de Fin de Grado en el Grado de Lingüística y Lenguas Aplicadas de la Universidad de Cádiz. Education in the Knowledge Society, 21, 1-10.

Eines, M., Aranda, N. y Amilivia, L. A. (2018). Las píldoras de contenidos. Revista Iberoamericana de Tecnología en Educación y Educación en Tecnología.21, 15-23.

Guevara, R. M., Sánchez-Moro, E. y Barrientos, G. (2020). Flipped Classroom y trabajo cooperativo en la Enseñanza Superior. En Redine (Ed.) Conference proceedings CIVINEDU 2020: 4th International Virtual Conference on Educational Research and Innovation (pp. 331-332). Madrid: Adaya Press.

Morán, C. y Fínez, M. J. (2019). Psicología del trabajo. Competencias para los Recursos Humanos. León: Eolas.

Muñoz, J. M., Espiñeira, E. V. y Rebollo, N. (2016). Las píldoras formativas: diseño y desarrollo de un modelo de evaluación en el Espacio Europeo de Educación Superior Revista de Investigación en Educación, 1(2), 156169. 
Rodríguez-García, A., Hinojo-Lucena, F. y Ágreda-Montoro, M. (2019). Diseño e implementación de una experiencia para trabajar la interculturalidad en Educación Infantil a través de realidad aumentada y códigos QR. Educar, 55(1), 59-77.

Salinas, J. y Marín, V. I. (2014). Pasado, presente y futuro del microlearning como estrategia para el desarrollo profesional. Campus Virtuales, 3(2), 46-61.

Sánchez-González, M. (2012). El "Acceso Abierto" como fórmula hacia una Universidad más adaptada al contexto de cultura digital: tendencias y experiencias en el caso español. Estudios sobre el Mensaje Periodístico, 18, 859-868.

Woods, K. (2020). The development and design of an interactive digital training resource for personal tutors. Frontiers in Education, Volume 5, Article 100.

Yokoyama, S. (2019) Academic Self-Efficacy and Academic Performance in Online Learning: A Mini Review. Frontiers in Education. Volume 9, Article 2794. 
